\title{
LISTA DE ESTIGOFAUNA CONOCIDA EN LA COMUNIDAD DE MADRID
}

\author{
A. I. Camacho* y A. G. Valdecasas*
}

\begin{abstract}
RESUMEN
El conocimiento de la estigofauna de la Comunidad de Madrid es muy escaso debido a que ha recibido muy poca atención por parte de los investigadores. Se conocen muy pocos datos que proceden de muestreos esporádicos y puntuales realizados, en la mayor parte de los casos, con objetivos taxonómicos. En este trabajo hemos intentado recoger toda la información publicada acerca de las especies de invertebrados acuáticos subterráneos, en sentido amplio, encontrados en cuevas, pozos artificiales, surgencias y medio intersticial asociado a ríos de la Comunidad de Madrid.
\end{abstract}

Palabras clave: estigofauna, Comunidad de Madrid, España.

\begin{abstract}
Comunidad de Madrid (Spain) stygobiotic fauna

Knowledge of Comunidad de Madrid stygofauna is very scarce due to the fact that very few researchers have paid attention to it. The data comes from sporadic sampling, mainly with a taxonomic purpose. In this paper we include all taxonomic information available on subterranean aquatic invertebrates, found in caves, artificial wells and interstitial environment associated with streams of Comunidad de Madrid.
\end{abstract}

Keywords: stygofauna, Comunidad de Madrid, Spain.

\section{Introducción}

Con el nombre de fauna estigobia se entiende el conjunto de animales, invertebrados acuáticos subterráneos, que viven en el agua que rellena los espacios que quedan entre los granos de arena del sedimento de las capas freáticas de ríos epigeos e hipogeos, de acuíferos, y de diferentes cuerpos de agua (gour, charcos, pozas, lagos, sifones, etc) de cuevas.
Estos medios resultan de difícil acceso y requieren técnicas de muestreo específicas, poco conocidas y en la mayoría de los casos de fabricación artesanal, para poder recoger la fauna que los habita, cuyo tamaño oscila entre $0,5 \mathrm{~mm}$ y llega hasta los 3-4 centímetros en casos excepcionales. La combinación de estos factores ha hecho que, en España, se hayan hecho pocas investigaciones sobre estos animales. En concreto, en la Comunidad de Madrid hemos podido recopilar

* Departamento de Biodiversidad y Biología Evolutiva. Museo Nacional de Ciencias Naturales de Madrid, CSIC. C/ José Gutiérrez Abascal 2. 28006- Madrid (España). e-mail: mcnac22@mncn.csic.es 
datos de estigofauna procedente de una cueva, de varios pozos artificiales, de una surgencia y del medio intersticial asociado a varios ríos y arroyos epigeos.

Los muestreos de los que proceden estos datos han sido realizados, casi siempre, con fines taxonómicos. Este es el caso de los muestreos llevados a cabo por Valdecasas (1981) para estudiar la fauna de Hydrachnidia de la Sierra de Guadarrama, de Camacho (1987) para estudiar la fauna de Syncarida en España y de Notemboom y Meijiers (1985) para estudiar Isopoda y Amphipoda estigobios de nuestra Península. En algunos de estos muestreos, no obstante, se ha tenido en consideración al resto de grupos faunísticos presentes y en algunos casos los ejemplares han sido determinados por especialistas; esto ocurre con Oligochaeta y con algunos Copepoda que han podido ser identificados a nivel de especie años después de ser recolectados (información inédita en muchos casos).

En el listado que ofrecemos a continuación aparece la fauna estrictamente estigobia marcada con un asterisco, ya que muchas de las especies encontradas en estos medios subterráneos viven también en medios acuáticos superficiales, siendo su presencia subterránea ocasional o más o menos frecuente según los casos y la época del año.

Dentro de cada grupo, la lista de especies es alfabética. Hemos evitado seguir una ordenación taxonómica para facilitar la búsqueda a los no especialistas en estos grupos faunísticos.

\section{Lista}

\section{TURbellaria Tricladida}

Atrioplanaria racovitzai (De Beauchamp 1928) Río Henares, Alcala de Henares, UTM: 30TVL .

Dugesia polychroa (Schmidt, 1861)

Río Henares, Alcala de Henares, UTM: 30TVL .

\section{Oligochaeta}

Marionina argentea (Michaelsen, 1889)

Cueva del Reguerillo, Cerro de la Oliva, Patones. UTM: 30TVL5824.

Aeolosoma leidyi Cragin, 1887

Cueva del Reguerillo, Cerro de la Oliva, Patones. UTM: 30TVL5824.

Cernosvitoviella atrata (Brestscher, 1903)

Cueva del Reguerillo, Cerro de la Oliva, Patones. UTM: 30TVL5824.

\section{Crustacea}

Attheyella (Attheyella) crassa (Sars, 1863) (Copepoda Harpacticoida)

Cueva del Reguerillo, Cerro de la Oliva, Patones. UTM: 30 TVL5824.

Bryocamptus (Rheocamptus) sp. gr. zschokkei (Schmeil, 1893) (Copepoda Harpacticoida)

Cueva del Reguerillo, Cerro de la Oliva, Patones. UTM: 30 TVL5824.

Bryocamptus (Bryocamptus) minutus (Claus, 1863)

(Copepoda, Harpacticoida)

Arroyo en el Puerto de la Morcuera, UTM: 30TVL296205.

*Canthocamptus staphylinus (Jurine, 1820) (Copepoda, Harpacticoida)

Río Jarama, Talamanca del Jarama, UTM: 30TVL5908.

Elaphoidella bidens (Schmeil, 1894) (Copepoda, Harpacticoida)

Río Jarama, Talamanca del Jarama, UTM: 30TVL5908.

Acanthocyclops robustus (Sars, 1863) (Copepoda, Cyclopoida)

Río Jarama, Talamanca del Jarama, UTM: 30TVL5908.

Diacyclops bisetosus (Rehberg, 1880) (Copepoda, Cyclopoida)

Río Jarama, Torrelaguna, UTM: 30TVL5219; Arroyo en el Puerto de la Morcuera, UTM: 30TVL296205.

Diacyclops sp.gr. crassicaudis Sars, 1863 (Copepoda, Cyclopoida)

Pozo 3, Valdetorres del Jarama, UTM: 30TVL59.

Paracyclops fimbriatus (Fischer, 1853) (Copepoda, Cyclopoida)

Río Jarama, Talamanca del Jarama, UTM: 30TVL5908;

Río Jarama, Torrelaguna, UTM: 30TVL5219; Surgencia en la

Cabrera; Arroyo en el Puerto de la Morcuera, UTM: 30TVL296205.

Eucyclops serrulatus (Fischer, 1853) (Copepoda, Cyclopoida)

Río Jarama, Talamanca del Jarama, UTM: 30TVL5908; Río Jarama, Torrelaguna, UTM: 30TVL5219; Pozo 1, Talamanca del Jarama, UTM: 30TVL59.

Megacyclops brachypus (Jurine, 1820) (Copepoda, Cyclopoida)

Pozo 2, Talamanca del Jarama, UTM: 30TVL59; Pozo 3, Valdetorres del Jarama, UTM: 30TVL59; Pozo 8; Valdetorres del Jarama, UTM: 30TVL59.

Megacyclops viridis (Jurine, 1820) (Copepoda, Cyclopoida)

Río Jarama. Pontón de la Oliva, UTM: 30TVL5824. 
*Hexaiberobathynella mateusi (Galhano, 1967), (Syncarida Bathynellacea)

Cueva del Reguerillo, Cerro de la Oliva, Patones, UTM: 30TVL5824; Río Jarama, Talamanca del Jarama, UTM: 30TVL5908; Río Jarama, Torrelaguna, UTM: 30TVL5219.

* Hexabathynella nicoleiana Camacho, 1986 (Syncarida Bathynellacea)

Río Jarama, Talamanca del Jarama, UTM: 30TVL5908; Río Jarama, Torrelaguna, UTM: 30TVL5219.

* Bathynellidae sp 1. (Syncarida Bathynellacea) Río Jarama. Pontón de la Oliva, UTM: 30TVL5824.

* Bathynellidae sp 2. (Syncarida Bathynellacea) Río en la Poveda.

* Bogidiella (Bogidiella) convexa Stock \& Notenboom, 1988 (Amphipoda)

Río Jarama, Talamanca del Jarama, UTM: 30TVL5908; Río Jarama, Torrelaguna, UTM: 30TVL5219.

\section{ACARI}

Arrenurus haplurus Viets, 1925 (Hydracnidia)

Arroyo de la Solana, antes de la Acebeda, Sierra de Guadarrama, UTM: 30TVL483483.

*Chappuisides hungaricus Szalay, 1943 (Hydracnidia) Río Moros, Estación del Espinar, Sierra de Guadarrama, UTM: 30TVL027119.

Feltria rouxi Walter, 1907 (Hydracnidia)

Río Lozoya a 2Km de Rascafría, UTM: 30TVL257274; surgencia en el camino de la Horizontal, pasada la Acebeda, UTM: 30TVL440484; primer arroyo después del Puerto de Navafria, UTM: 30TVL382.

Frontipodopsis reticulatifrons Szaalay, 1945 (Hydracnidia) Arroyo de la Solana, antes de la Acebeda, UTM: 30TVL483483; surgencia en el camino de la Horizontal, pasada la Acebeda, UTM: 30TVL440484; tercer arroyo en la carretera de Lozoya a Navafría, UTM: 30TVL312367; arroyo de la Barranca, La Barranca, UTM: 30TVL162138.

*Hungarohydracarus subterraneus Szalay, 1943 (Hydracnidia)

Río Eresma, Navacerrada, UTM: 30TVL141248.

Lethaxona pygmaea Viets, 1932 (Hydracnidia)

Río Manzanares, Madrid (i?) (localidad 1 en Viets, 1930); río Manzanares, Puente de San Fernando (¿?) (localidad 7 en Viets, 1930); charca de Marmoto, Manzanares El Real (i?) (localidad 15 en Viets, 1930).

*Lobohalacarus weberi quadriporis (Walter, 1947) (Halacaridae)

Intersticial arroyo Salino, Aranjuez, UTM: 30TVK4330; intersticial arroyo de la Barranca, La Barranca, UTM: 30TVL162138.
Protzia invalvaris Piersig, 1898 (Hydracnidia)

Río Lozoya entre Navacerrada y el Paular, a $12 \mathrm{Km}$ del Paular, UTM: 30TVL212211; arroyo de la Solana, antes de la Acebeda, UTM: 30TVL483483; arroyo de la Barranca, La Barranca, UTM: 30TVL162138.

Pseudotorrenticola rhynchota Walter, 1906 (Hydracnidia) Musgos arroyo, $1200 \mathrm{~m}$. Madrid (i?) (Lundblad, 1957); arroyo de la Solana, antes de la Acebeda, UTM: 30TVL483483; arroyo de la Barranca, La Barranca, UTM: 30TVL162138; primer arroyo después del Puerto de Navafría, UTM: 30TVL382.

*Stygomomonia latipes Szalay, 1943 (Hydracnidia)

Río Moros, Estación del Espinar (Segovia), UTM: 30TVL027119.

*Wandesia thori Schechtel, 1912 (Hydracnidia)

Arroyo de la Barranca, La Barranca, UTM: 30TVL162138; primer afluente del arroyo de los Acebos, La Fuenfría, UTM: 30TVL091146; arroyo al lado del Chalet de Peñalara, UTM: 30TVL101153.

\section{AGRADECIMIENTOS}

Queremos dar las gracias a M. L. Peláez y a A. Correas que nos han ayudado de diferentes maneras. La Comunidad de Madrid ha hecho posible la realización de este trabajo con su aportación económica a través del proyecto GR/AMB/0750/2004 concedido al Museo Nacional de Ciencias Naturales (CSIC).

\section{Referencias}

Bellés, X., 1987. Fauna Cavernicola i Intersticial de la Peninsula Iberica $i$ les Illes Balears. Monografies Cientifiques, 4, CSIC, Editorial Moll, Mallorca. 207 pp.

Bello, E., 1996. Herramientas taxonómicas por ordenador: los halacáridos subterráneos continentales en la Península Ibérica. Tesis Doctoral, Universidad Autónoma de Madrid. 510 pp.

Camacho, A. I., 1986. A new species of the genus Hexabathynella (Syncarida, Bathynellacea, Parabathynellidae) from Spain. Bijdrajen tot Dierkunde, 56(1): 123-131.

Cамасно, A. I., 1987. La familia Parabathynellidae (Crustacea, Syncarida, Bathynellacea) en la Península Ibérica. Taxonomía, Filogenia y Biogeografia. Tesis Doctoral, Universidad Autónoma de Madrid, 889 pp.

CAmacho, A. I., 1989. Los Batineláceos (Crustacea, Syncarida) recogidos en la Península Ibérica. Boletín de la Asociación Española de Entomología, 13: 71-90.

CAMACHO, A. I., 2003. An overview of the Distribution of the Parabathynellidae family (Crustacea, Syncarida, Bathynellacea) on the Iberian Peninsula. Graellsia, 59(1): 63-78. 
Camacho, A. I. \& Serban, E., 1998. Tribu des Iberobathynellini nov. Diagnoses des taxa nouveaux (Podophallocarida, Bathynellacea, Parabathynellidae). Travaux de l'Institute de Speologie Emile Racovitza, [1985], 34: 15-75.

Camacho, A. I., Serban, E. \& Guil, N., 2000. Phylogenetical review and biogeographic remarks on the interstitial and subterranean freshwater iberobathynells (Crustacea, Syncarida, Parabathynellidae). Journal of Natural History, 34: 563-585.

LuNDBLAD, O., 1957. Zur Kenntnis süd- und mitteleuropäicher Hydrachnellen. Arkiv för Zoologi, 10(1): 1306.

Notenboon, J. \& MeIJERS, I., 1985. Investigaciones sobre la fauna de las aguas subterráneas en España: Lista de estaciones y primeros resultados (Research on the groundwater fauna of Spain: List of stations and first results). Verslagen en Technische Gegevens, 42, Instituut voor Taxonomische Zoölogie-Plantage Middenlaan 53. Amsterdam. 93 pp.
VALDECASAS, A. G., 1981. Las hidracnelas de la sierra del Guadarrama: Taxonomía, distribución y ecología. Tesis Doctoral, Universidad Complutense de Madrid. $532 \mathrm{pp}$.

VALDECASAS, A. G., 1988. Lista sinonímica y bibliográfica de las hidracnelas (Acari, Hydrachnellae) de la Península Ibérica, Islas Baleares e Islas Canarias. Listas de la Flora y Fauna de las Aguas Continentales de la Peninsula Iberica ${ }^{\circ} 5$. Asociación Española de Limnología. Madrid. $81 \mathrm{pp}$.

VIETS, K., 1930. Zur kenntnis dert Hydracarinen-Fauna von Spanien. Archiv für Hydrobiologie, 21: 175-240; $359-446$. 